\title{
Reference patterns and the process of normalization
}

\author{
SEBASTIANO BAGNARA \\ Institute of Psychology, National Research Council, Roma, Italy \\ FRANCESCA SIMION \\ Institute of Developmental Psychology, University of Padova, Padova, Italy \\ and \\ CARLO UMILTÀ \\ Institute of Human Physiology, University of Parma, Parma, Italy
}

\begin{abstract}
Two experiments investigated the effect of reference patterns on the normalization process that involves the visual code. In Experiment 1, the subjects had to classify as same or different two simultaneously presented letters. The upper letter was always in the upright position, whereas the lower letter could take six different orientations and was enclosed in a congruent triangular frame. The results for same responses showed reliable orientation-dependent effects which clearly implied an operation of normalization. In Experiment 2, the orientation of the upper letter and that of its frame varied orthogonally, whereas the lower letter was always presented within a congruent frame at six different orientations. The results showed that the normalization occurred with reference to the orientation of the upper letter, and that the congruent upper frame could speed up the comparison process only at certain orientations of the lower letter. It was concluded that the direction of normalization depends on the orientation of the upper letter, and that the effect of the frame is interpretable in terms of a display symmetry facilitation effect.
\end{abstract}

Human observers are faster in classifying two visual patterns as same when the two patterns share the same orientation than when they do not. It is clear that an extra operation is needed to correctly classify two disoriented patterns and that this is the cause of the slower response latencies. What is still in dispute is the nature of such an extra operation.

The most widely accepted hypothesis is that of a nori.ualization process that precedes the comparison stage. Observers could perform some sort of visual transformation that brings patterns into spatial congruence. Within the framework of this hypothesis, two mechanisms can be distinguished. In some circumstances, the normalization involves the rotation of an internally generated visual image (see, e.g., review in Shepard, 1975). In other circumstances, the operation of normalization concerns the visual code (Posner, 1978; Simion, Bagnara, Roncato, \& Umiltà, 1982).

Some of the results reported here were preliminarily communicated at the 25th Annual Meeting of the Human Factors Society, Rochester (NY), 1981. The authors are grateful to Sandro Bettella for help in preparing the stimulus material and to Sergio Roncato for valuable suggestions. Requests for reprints should be sent to Sebastiano Bagnara, Institute of Psychology, National Research Council, Via dei Monti Tiburtini, 509, 00157 Roma, Italy.
The distinction between a mental image and the visual code originated with Shepard (1975) and was further elaborated by Posner (1978). Visual image refers to an internal representation that is accessible to the observer's introspection and takes time to be generated. The visual code consists of an automatic priming of the visual feature detectors. The operations of normalization upon the visual code are achieved automatically through an effortless process that does not require clear awareness by the observer. Hence, they are faster than operations upon a visual image which, by contrast, demand attention and are accompanied by subjective reports of imagery.

The possibility that internally generated visual images can be rotated mentally has been extensively corroborated by Shepard and his colleagues (see, e.g., Cooper \& Shepard, 1973, and review in Shepard, 1975, and Shepard \& Cooper, 1982). Typically, the observer is asked to form an image of an absent pattern which has to be matched with another pattern that will be presented as soon as the requested image is formed. In such a task, response latencies increase monotonically as the visual pattern deviates from the orientation of the image.

In a same-different classification task, when both patterns are physically present, it is assumed that the 
observer forms and maintains a visual image of one for comparison with the other (Shepard, 1975). However, this notion is tenable only if the observer is allowed enough time (1-2 sec) to generate an image of sufficient clarity. It cannot be invoked to explain the orientation-dependent effects that are observed in speeded classification tasks when the two patterns to be compared are simultaneously present. In fact, it has been claimed that in these circumstances the operation of normalization applies to the visual code.

In a recent series of experiments, Simion et al. (1982) clarified the characteristics of the operations of normalization based on the visual code. Their main findings can be summarized as follows. First, there was no orientation effect when the observers correctly classified two different letters. Second, even though reliable orientation effects could be observed in the case of same responses, the rate of normalization was an order of magnitude higher than that typical of rotation of visual images (about $2,500 \mathrm{deg} / \mathrm{sec}$ vs. about $300 \mathrm{deg} / \mathrm{sec}$ ). Third, it was found that frames of reference that jointly rotate with the disoriented letters could eliminate any effect of orientation even for same responses. Overall, the results showed that the characteristics of the normalization process that applies to the visual code are different from those observed for visual images.

In that study, the subjects had to classify as same or different two simultaneously presented letters placed one above the other. In three experiments, the upper letter was centered in a frame and both the letter and the frame were in the upright orientation. The lower letter, the orientation of which could vary from 0 to 300 deg in 60-deg steps, was centered in a frame identical to that enclosing the upper letter, and the vertical axes of the letter and the frame coincided. It was apparent that the rotating frames could eliminate the increase in response latencies due to the difference in orientation between the two letters.

No conclusive explanation was proposed to account for the effect of the frame. However, it was shown that the frame did not act as a directional cue, because an arrowhead that gave explicit directional information could not eliminate the orientation effects. It was also shown that such effects could not be prevented by simply enclosing the two letters in a fixed frame. Thus, what seemed to be instrumental in eliminating the effect of the difference in orientation was the invariance of the spatial relations between the enclosing pattern and the enclosed letter. If this is the case, the orientation effect should become apparent again when only one of the two letters is enclosed in a frame. This is because in such a condition the relations at the border between one of the two letters and its frame cannot be utilized for performing the comparison. The goal of Experiment 1 was to gather empirical evidence to support this hypothesis.

\section{EXPERIMENT 1}

Experiment 1 was very similar to Experiments 4, 5, and 6 of Simion et al. (1982) which had demonstrated the effect of the frame. The only notable exception was that in the present experiment the upper letter was presented without any frame. It is clear that when two letters are presented simultaneously it becomes difficult to determine which one acts as the criterion reference pattern. However, it would seem reasonable to assume that in the conditions of the present study the criterion letter was the upper one. This is because its orientation remained unchanged within each block of trials, and, as suggested by Ambler \& Proctor (1976), an observer first processes the top half of a visual display.

\section{Method}

Subjects. Twelve students ( 6 males and 6 females), ranging in age between 19 and 25 years, took part in the experiment. They had normal or corrected-to-normal vision. They were paid for their collaboration.

Stimuli. The stimuli (see Figure 1) consisted of pairs of black uppercase forms of $F, G_{3}$ and $R$ (taken from MECANORMA 22 28.C) on white background. A photographic negative (35 mm) of each pattern was mounted in a slide holder for tachistoscopic projection on a screen. The letters were placed vertically. The upper letter always appeared in the upright position (0-deg orientation). The lower letter had six angular orientations, from 0 to 300 deg in 60-deg steps of clockwise rotation, and was always enclosed in a congruent triangular frame (i.e., the top of the enclosing triangle was always at the top of the enclosed letter). At the viewing distance adopted throughout the experiment, each letter subtended a visual angle of $1.7 \mathrm{deg}$ in the upright position. The base and height of the triangular frame subtended 3.4 and $4.6 \mathrm{deg}$. The distance between the vertex of the triangle (when it was at 0 deg) and the base of the upper letter was 1.1 deg.

Procedure. The subject sat at a distance of $60 \mathrm{~cm}$ from a tangent translucent screen, with his or her head positioned in a head-and chinrest. An acoustic signal prompted him/her to fixate the place on the screen where the stimuli were to be presented. Half a second later, a slide was back-projected for $100 \mathrm{msec}$. The interval between two successive presentations was $5 \mathrm{sec}$.

Stimulus intensity was $45 \mathrm{~cd} / \mathrm{m}^{2}$, and the luminance of the ambient light and fixation field was 21 and $20 \mathrm{~cd} / \mathrm{m}^{2}$, respectively. The subject was instructed to respond by pressing one of two keys
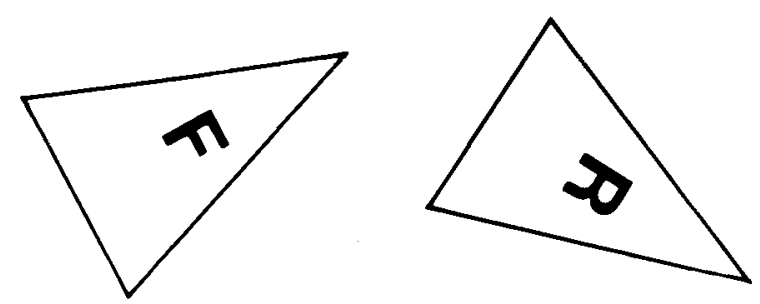

Figure 1. Examples of the stimali employed in Experiment 1. 
with the right or left index finger as quickly as possible and try to avoid errors. The keys were on a response panel positioned just below the center of the screen. Half of the subjects $(3$ males and 3 females) used the right hand for same responses and the left hand for different responses; the other subjects had the reverse assignment. Pressing the key stopped one of two electronic millisecond counters that were started at the beginning of the $100-\mathrm{msec}$ exposure period.

There were three possible same pairs, and they were presented five times each in the six orientations, bringing the number of same stimuli to 90 . Thirty-six different pairs were obtained by pairing each letter with one of the other two in every orientation. Since each different pair was presented twice, 72 different stimuli were shown.

The subjects were tested in one session that lasted about $60 \mathrm{~min}$. Formal testing began after 80 practice trials. The data-collecton session consisted of 162 experimental trials divided into two blocks separated by a S-min rest period.

The stimuli were presented in a random sequence. No feedback on speed or accuracy was given to the subjects, but error trials were repeated at the end of the session. The subjects who exceeded a limit of eight errors in the 162 trials (i.e., $5 \%$ ) were excluded from the experiment. As a result, two subjects had to be replaced.

\section{Results}

Reaction time (RT) data for same responses were entered into two one-way repeated-measures analyses of variance. In the first analysis, because the results for the upright orientation were included twice, there were seven levels of orientation: $0,60,120,180,240$, 300 , and $360 \mathrm{deg}$. In the second analysis, the results for the upright orientation were excluded, and there were five levels of orientation: $60,120,180,240$, and 300 deg. This latter analysis was carried out because previous studies had shown that the upright orientation yielded the fastest RTs independently of any operation of normalization (see, e.g., Ambler \& Proctor, 1976; Egeth \& Blecker, 1971; Kolers \& Perkins, 1969; Simion et al., 1982). It was expected that the normalization process, if present, would manifest itself even when the upright orientation was excluded. Both analyses demonstrated a significant effect of orientation $[F(6,66)=7.82, p<.001$, and $F(4,44)=4.84$, $\mathrm{p}<.005$, respectively]. Response latencies varied as a function of the orientation of the comparison letter: $571,608,617,634,584$, and $601 \mathrm{msec}$ for the 0-, 60-, $120-, 180-, 240-$, and 300-deg orientations, respectively. Further analyses were performed to test linear, quadratic, cubic, and quartic trends with the level of significance set at $\alpha=.01$. Only the quadratic trends were significant $[F(1,15)=19.63, p<.001$, with the 0 -deg data, and $F(1,15)=8.71, p<.01$, without the 0-deg data].

Similar analyses were conducted on RTs for different responses, and no significant effect of orientation was found. The mean response latencies were 641 , $666,665,667,665$, and $648 \mathrm{msec}$ for $0,60,120,180$, 240 , and $300 \mathrm{deg}$, respectively.

Because of the high requirements set for accuracy, the errors were very few (less than 2\%) and were not submitted to statistical analysis.

\section{Discussion}

First of all, it must be stressed that the rate of normalization ${ }^{1}$ was extremely high $(2,223 \mathrm{deg} / \mathrm{sec})$. This indicated the visual code as the internal representation involved in the operation of normalization.

In a previous study (Simion et al., 1982), we had found that frames that were congruent with the two letters could eliminate the effects of orientation. This finding can be explained by assuming that the subjects made use of the invariant spatial relationships present at the border between the enclosing frame and the enclosed letter. By following this line of reasoning, we suggested that, because those spatial relationships could no longer be utilized for a correct classification, the effects of orientation should become manifest again when only one of the two letters to be compared was enclosed in a congruent frame. The results of the present experiment gave empirical support to such a hypothesis by showing significant effects attributable to the difference in orientation between the upper and the lower letter.

However, a different interpretation can be put forward to explain the facilitatory effect of the congruent frame. It is known (see Bagnara, Boles, Simion \& Umiltà, 1983; Kahn \& Foster, 1981; Richards, 1978) that the whole display symmetry can speed up the comparison process that underlies same responses. As a matter of fact, in our previous study (Simion et al., 1982) the disappearance of the quadratic trend taken as indicative of a normalization process was due to the relatively faster responses observed when the lower letter and its frame were at $180 \mathrm{deg}$. In other words, the congruence effect was strongest at a position that yielded the whole display symmetry along the horizontal axis. These two interpretations can be better tested by varying the orientations of the upper letter and frame. The interpretation in terms of invariant border relationships predicts that the congruence effect should manifest itself irrespective of the relative position of the upper and lower letters. In contrast, the interpretation in terms of whole display symmetry predicts that the congruence effects should be present at only those orientations that produce such symmetry. The following experiment was also aimed at clarifying this issue.

The foregoing discussion does not apply to different responses. In fact, when the subjects correctly classified two different letters, no evidence of an effect of orientation was found. This is a typical finding of those studies in which the operations of normalization are based on the visual code (Besner \& Coltheart, 1975, 1976; Kubovy \& Podgorny, 1981; Santee \& Egeth, 1980; Simion et al., 1982). The explanation that is usually offered to account for the discrepancy between the two types of response makes reference to two different processors. The holistic processor, which mediates same responses, would be 
sensitive to disparities along an irrelevant dimension (orientation in the present experiment), whereas the analytic processor, which mediates different responses, would not. Hence, only same responses would require an operation of normalization before a correct classification is accomplished.

\section{EXPERIMENT 2}

In all the experiments mentioned so far, the upper letter and its frame were always in the upright position. In the following experiment, the orientation of the upper letter and that of the upper frame could have two values that were varied orthogonally. Such an experimental condition seemed appropriate for clarifying the following points.

The first point was similar to that raised by Rolden and Phillips (1980) in relation to the normalization processes based on visual images. These authors found differential effects on the normalization process as a function of whether the imaged criterion was in the upright or in the upside-down position: In the first case, RTs increased monotonically with the angular distance between the image and the test pattern, whereas in the second case, the RTs did not increase monotonically. Experiment 2 aimed at establishing whether similar effects were to be found when the visual code was the internal representation on which normalization took place.

The second point was aimed at discriminating between the two above hypotheses concerning the congruence effect. As already pointed out, according to the hypothesis of the invariant border relationships, the effect should be always present, whereas according to the symmetry hypothesis, it should be confined at certain orientations of the upper and lower letters.

Finally, we tried to disentangle the effects on the normalization process of the orientation of an upper letter and that of an upper frame. In the previous experiments (Simion et al., 1982), these two factors were confounded because the frame was always congruent with the upper letter.

\section{Method}

The present experiment actually consisted of two distinct parts. In Part A, the upper letter and the upper frame were presented at either 0 or 120 deg of clockwise (CW) rotation, whereas in Part $B$, the orientations employed were 0 and $240 \mathrm{deg}$, that is, $120 \mathrm{deg}$ of counterclockwise (CCW) rotation. In both parts, the two factors (i.e., orientation of the upper letter and orientation of the upper frame) varied independently and orthogonally so that, for each direction of rotation ( $\mathrm{CW}$ and $\mathrm{CCW}$ ), there were four experimental conditions: (1) letter at $0 \mathrm{deg}$ and frame at $0 \mathrm{deg}$, (2) letter at $0 \mathrm{deg}$ and frame at $120 \mathrm{deg}$, (3) letter at $120 \mathrm{deg}$ and frame at $0 \mathrm{deg}$, and (4) letter at $120 \mathrm{deg}$ and frame at $120 \mathrm{deg}$. As in Experiment 1, the lower letter was always enclosed in a congruent frame and was presented at six different orientations (i.e., $0,60,120,180,240$, and 300 deg).

Subjects. Thirty-two students (16 males and 16 females) in the age range between 19 and 25 years received a small fee for participating in the experiment. All were right-handed, had normal or

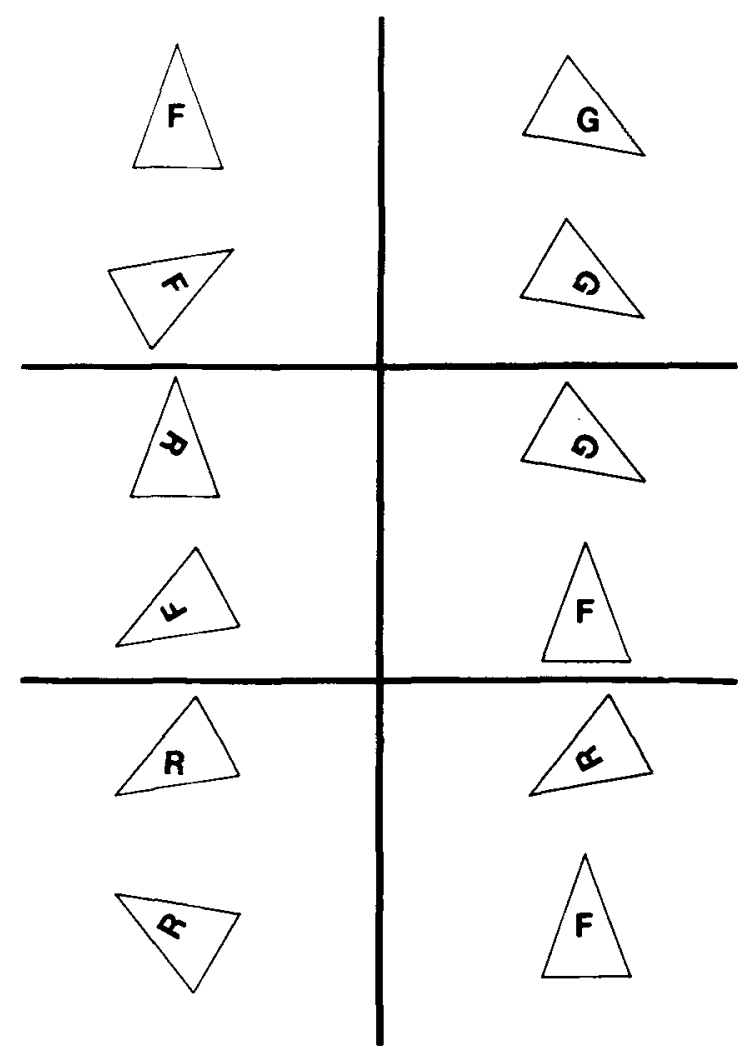

Figure 2. Examples of the stimuli employed in Experiment 2.

corrected-to-normal vision, and had not taken part in Experiment 1 . The subjects served in four consecutive daily sessions, 16 ( 8 males and 8 females) in Part A, and 16 in Part $B$.

Stimull. The stimuli (see Figure 2) were constructed in the same way as those of Experiment 1. The comparison (lower) letters exactly replicated those previously described. The criterion (upper) letters could be shown at 0 or $120 \mathrm{deg}$ with either $\mathrm{CW}$ (Part A) or CCW (Part B) rotation, and were enclosed in a triangular frame which would independently take the same orientations. The triangular frame was identical to that employed for the lower letters. Thus, while the frame of the lower letters was always congruent, that of the upper letters could be either congruent or incongruent.

The size of the stimuli was the same as in Experiment 1 . In the upright position, the upper and the lower letters along with their frames subtended a visual angle of $10.3 \mathrm{deg}$, while the distance between the vertex of the lower triangle and the base of the upper one (when both at 0 deg) was 1.1 deg.

Procedure. The procedure exactly replicated that described for Experiment 1 with the following exceptions. For both parts there were four data-collection sessions, defined by the relative positions of the upper letter and frame, and every subject was presented with all the experimental conditions in four successive sessions. One session lasted about $60 \mathrm{~min}$ and comprised 162 trials ( 90 for same pairs and 72 for different pairs). The order of presentation of the four experimental conditions was balanced across subjects. Also, in the present experiment the limit for accuracy was set at $5 \%$ of errors, and since the errors were fewer than $2 \%$, they were not analyzed.

\section{Results}

The RT data for same responses were submitted to two four-way analyses of variance. The only betweensubjects factor was direction of rotation (CW or CCW). Of course, a CW direction denotes Part A 
Table 1

Mean Reaction Times (RTs) (in Milliseconds) for Same and Different Responses in Experiment 2 as a Function of the Orientation (in Degrees) of the Upper Letter and of the Lower Letter

\begin{tabular}{|c|c|c|c|c|c|c|c|}
\hline \multirow{2}{*}{$\begin{array}{r}\text { Type of } \\
\text { Response }\end{array}$} & \multirow{2}{*}{$\begin{array}{c}\text { Orientation of the } \\
\text { Upper Letter }\end{array}$} & \multicolumn{6}{|c|}{ Orientation of the Lower Letter } \\
\hline & & 0 & 60 & 120 & 180 & 240 & 300 \\
\hline \multirow[t]{2}{*}{ Same } & $\begin{array}{l}0 \\
120\end{array}$ & $\begin{array}{l}510 \\
544\end{array}$ & $\begin{array}{l}530 \\
532\end{array}$ & $\begin{array}{l}539 \\
512\end{array}$ & $\begin{array}{l}554 \\
548\end{array}$ & $\begin{array}{l}534 \\
547\end{array}$ & $\begin{array}{l}541 \\
560\end{array}$ \\
\hline & $\mathbf{M}$ & 527 & 531 & 526 & 551 & 540 & 550 \\
\hline \multirow[t]{2}{*}{ Different } & $\begin{array}{l}0 \\
120\end{array}$ & $\begin{array}{l}572 \\
586\end{array}$ & $\begin{array}{l}575 \\
602\end{array}$ & $\begin{array}{l}588 \\
603\end{array}$ & $\begin{array}{l}580 \\
586\end{array}$ & $\begin{array}{l}588 \\
593\end{array}$ & $\begin{array}{l}580 \\
576\end{array}$ \\
\hline & $\mathbf{M}$ & 579 & 588 & 596 & 583 & 590 & 578 \\
\hline
\end{tabular}

and a CCW direction denotes Part B of the experiment. The three within-subjects factors were: orientations of the upper frame ( 0 or $120 \mathrm{deg})$ and orientation of the lower letter and its frame $(0,60,120,180$, 240,300 , and $360 \mathrm{deg}$ ).

There was a significant main effect of the orientation of the lower letter $[F(6,180)=22.09, p<.001]$ and a significant interaction of orientation of the upper letter $X$ orientation of the lower letter $[F(6,180)=33.50, p<.001]$. The relevant data are shown in Table 1.

Simple main effects tests were carried out, followed by trend analyses. Both the simple main effects and the quadratic trends were significant [upper letter at $0 \mathrm{deg}, F(6,180)=19.93, \mathrm{p}<.001$, and quadratic trend, $F(1,30)=32.27, p<.001$; upper letter at $120 \mathrm{deg}, \mathrm{F}(6,180)=12.33, \mathrm{p}<.001$, and quadratic trend, $\mathrm{F}(1,30)=22.65, \mathrm{p}<.001]$.

Finally, there was a significant interaction involving orientation of the upper letter, orientation of the upper frame, and orientation of the lower letter $[F(6,180)=$ $3.26, \mathrm{p}<.005]$. The relevant data are reported in Table 2 .

The two-way interaction clearly showed that the fastest RTs were always those obtained when the two letters to be compared shared the same position ( 0 and $120 \mathrm{deg}$ ). The position itself did not play any important role since there was virtually no difference in RT between when the letters were both at 0 deg and when they were at $120 \mathrm{deg}$ (see Table 1). Furthermore, response latencies tended to increase in a similar fashion with the angular distance between the orientation of the upper letter and that of the lower letter, irrespective of the position of the former.
The three-way interaction is attributable to the fact that the congruent frame enclosing the upper letter had an effect at only some positions of the lower letter. This can be seen in Table 3, which shows the effect of the congruence of the upper frame with the upper letter at the various orientations of the lower letter. A negative figure indicates that response latencies were faster when the upper frame was congruent with the upper letter than when it was not. A positive value indicates the opposite effect. It seems clear that the congruent upper frame speeds up the comparison process mainly, if not exclusively, when the two letters shared the same orientation (i.e., 0 or $120 \mathrm{deg}$ ) or were positioned at symmetrical orientations (i.e., 0 and $180 \mathrm{deg}$, or 120 and $240 \mathrm{deg}$ ). The only discrepant result was that of a facilitatory effect for the lower letter at 0 deg when the upper one was at $120 \mathrm{deg}$. In any event, the congruence effect was always rather small, that is, on the order of $10-16 \mathrm{msec}$.

Analyses of variance with the same factors were carried out on different RTs. The main effect of orientation of the lower letter and its interaction with orientation of the upper letter were significant $[F(6,180)=7.75, p<.001$, and $F(6,180)=5.82$, $\mathrm{p}<.001$, respectively; see Table 1 for the relevant data]. The fact that this finding is at variance with a number of previous studies (Besner \& Coltheart, 1975, 1976; Kubovy \& Podgorny, 1981; Santee \& Egeth, 1980; Simion et al., 1982), however, it should not be accorded too much importance, since the lack of significant quadratic trends does not allow any interpretation in terms of an orderly process of normalization. Therefore, the results for different responses will not be discussed further.

Table 2

Mean Reaction Times (RTs) (in Milliseconds) for Same Responses in Experiment 2 as a Function of the Orientations (in Degrees) of the upper letter, the upper frame, and the lower letter

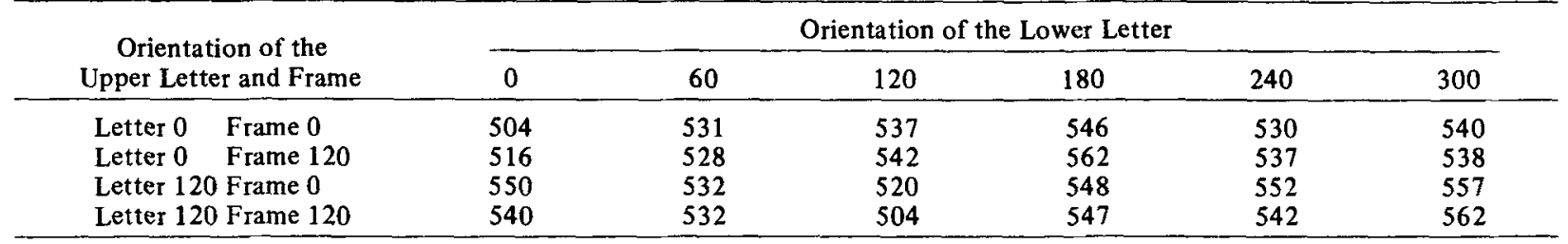


Table 3

Effect (in Milliseconds) of the Congruence of the Upper Frame with the Upper Letter as a Function of the Orientation (in Degrees) of the Lower Letter

\begin{tabular}{|c|c|c|c|c|c|c|c|c|c|c|c|c|}
\hline \multicolumn{13}{|c|}{ Orientation of the Lower Letter } \\
\hline & \multicolumn{6}{|c|}{ Upper Letter at 0} & \multicolumn{6}{|c|}{ Upper Letter at 120} \\
\hline & 0 & 60 & 120 & 180 & 240 & 300 & 0 & 60 & 120 & 180 & 240 & 300 \\
\hline Congruent Frame & 504 & 531 & 537 & 546 & 530 & 540 & 540 & 532 & 504 & 547 & 542 & 562 \\
\hline Noncongruent Frame & 516 & 528 & 542 & 562 & 537 & 538 & 550 & 532 & 520 & 548 & 552 & 557 \\
\hline Effect of Congruence & -12 & 3 & -5 & -16 & -7 & 2 & -10 & 0 & -16 & -1 & -10 & 5 \\
\hline
\end{tabular}

\section{Discussion}

The significant interactions between orientation of the upper letter and orientation of the lower letter showed that the normalization process took place with reference to the actual orientation of the former. The fastest RTs were observed when the lower and upper letters shared the same orientation (i.e., 0 and 120 deg), whereas the slowest were those for a lower letter positioned at the greatest $(180 \mathrm{deg})$ angular distance from the upper one (i.e., 180 and $300 \mathrm{deg}$ ).

It is also worth noting that the direction of rotation (i.e., $\mathrm{CW}$ or $\mathrm{CCW}$ ) did not affect the normalization process.

These results indicate that the reference orientation is defined according to the actual position of the upper letter. This outcome is consistent with a previous finding by Ambler and Proctor (1976), who had shown that the normalization process is not linked to the upright position. It also shows some analogies with what was found when visual images were presumably used (Roldan \& Phillips, 1980). With either visual images or the visual code, the normalization process seemed not to have a privileged reference position, but depended on where the criterion was actually presented or imaged.

However, some discrepancies indicate that no complete functional equivalence can be hypothesized between visual images and the visual code. First, the rate of normalization was very high in the present experiment: $2,625 \mathrm{deg} / \mathrm{sec}$ with the upper letter at $0 \mathrm{deg}$ and $1,825 \mathrm{deg} / \mathrm{sec}$ with the upper letter at $120 \mathrm{deg}$. These values are about nine times as large as those found by Roldan and Phillips (1980) for rotation of visual images. Second, there was not a clear advantage for matching two letters that were both in the standard upright position as compared to when they shared the same inclination. Finally, the process of normalization showed about the same characteristics independently of the position it has to be directed to. Such outcome is interesting because, in the case of very familiar stimuli, which have an overlearned standard orientation, one could predict that the normalization process should vary as a function of whether it has to be directed to the standard or to a nonstandard disoriented position.

The nonsignificant interactions between orientation of the upper frame and orientation of the lower letter showed that the frame in itself had no role in determining the characteristics of the processes preceding the matching of the two letters. This confirms what has been previously stated, that a frame can influence the processes only through its relations with the enclosed letter.

The interaction involving orientation of the upper letter, orientation of the upper frame, and orientation of the lower letter points to important limitations of the role of the frame in matching two disoriented letters, and gives support to the whole symmetry hypothesis. Based on the results of a previous study (Simion et al., 1982), it was concluded that a congruent frame eliminates orientation-dependent effects. It was also argued that the observer can utilize the invariant relationships present at the border between the letter and the frame in order to perform the comparison without a preceding process of normalization. Now, it seems apparent that a congruent frame affects the comparison process only at certain orientations. More specifically, the congruent frame facilitates the comparison process only when the upper and the lower letters and their frames are positioned in such a way as to yield a symmetrical display. Thus, it can be suggested that the congruence effects observed in the present and previous experiments (Simion et al., 1982) is due to the whole display symmetry that emerges at certain orientations and not to the invariant spatial relationships present at the border between the letter and its frame. This is not to say that the display symmetry is brought about by the triangular frames. It can no doubt also be present with pairs of letters without frames. However, it is conceivable that the frames can be instrumental in rendering the display symmetry more salient and thus easier to be utilized in the comparison process.

The acceptance of the symmetry hypothesis leads to the conclusion that the frame has no role in the normalization process. It merely renders faster some of the comparisons and thus can simulate a bypassing of the normalization process.

\section{CONCLUSION}

Some of the findings of the present experiments confirmed those of previous studies, but others were 
new. The former can be summarized as follows: (1) The process of normalization that occurs in a same-different classification task when the two stimuli are simultaneously present and the distractors have different shapes takes place on the basis of the visual code; (2) the time-course of this normalization process is markedly different from that of the rotation of a visual image; and (3) the presence of a congruent frame of reference which encloses the two letters affects the normalization process.

The new findings shed light on the characteristics of the process of normalization and on the role played by the frame in this context. It was found that (1) normalization is sensitive to the position of the pattern that determines the point of reference of the required transformation; and (2) the frame acts by rendering more salient the display symmetry present at certain orientations and thus speeding up some of the comparison processes that mediate same responses.

These findings suggest that when the visual code is the internal representation on which the comparison is performed, an observer may compare two disoriented patterns by bringing them into congruence through a very fast normalization process, which resembles that described as a mental rotation when visual images are involved. However, this normalization process shows clear differences when compared with a canonical mental rotation.

\section{REFERENCDS}

Amblen, B. A., \& Proctor, J. D. (1976). The familiarity effect for single letter pairs. Journal of Experimental Psychology: Human Perception and Performance, 2, 222-234.

Baqnara, S., Boles, D. B., Simion, F., \& Umiltà, C. (1983). Symmetry and similarity in lateralized letter matching. Perception \& Psychophysics, 34, 578-584.

Besner, D., \& Coltheart, M. (1975). Same-different judgments with words and nonwords: The differential effects of relative size. Memory \& Cognition, 3, 673-677.
Besner, D., \& Coltheart, M. (1976). Mental size scaling examined. Memory \& Cognition, 4, 525-531.

Coopen, L. A., \& Shepard, R. N. (1973). Mental rotation of letters. In W. G. Chase (Ed.), Visual information processing. New York: Academic Press.

Egeth, H., \& Blecken, R. (1971). Differential effects of familiarity on judgments of sameness and difference. Perception \& Psychophysics, 9, 321-326.

KahN, J. L., \& Foster, D. M. (1981). Visual comparison of rotated and reflected random-dot patterns as a function of their positional symmetry and separation in the field. Quarterly Journal of Experimental Psychology, 33, 155-165.

Kolens, P. A., \& Perkins, D. N. (1969). Orientation of letters and errors in their recognition. Perception \& Psychophysics, $5,265-269$.

Kubovy, M., \& Podgonny, P. (1981). Does pattern matching require the normalization of size and orientation? Perception \& Psychophysics, 30, 24-28.

Posner, M. I. (1978). Chronometric explorations of mind. Hillsdale, NJ: Erlbaum.

Richards, J. T. (1978). Interitem structure and the facilitation of simultaneous comparison. Journal of Experimental Psychology: Human Perception and Performance, 4, 72-87.

Roldan, C. E., \& Phillips, W. A. (1980). Functional differences between upright and rotated images. Quarterly Journal of $E x$ perimental Psychology, 32, 397-412.

Santee, J. L., \& Egeth, H. E. (1980). Selective attention in speeded classification and comparison of multidimensional stimuli. Perception \& Psychophysics, 28, 191-204.

ShEPARD, R. N. (1975). Form, formation, and transformation of internal representation. In R. Solso (Ed.), Information processing and cognition: The Loyola Symposium. Hillsdale, NJ: Erlbaum.

Shepard, R. N., \& Cooper, L. A. (1982). Mental images and their transformations. Cambridge, MA: M.I.T. Press.

Simion, F., Bagnara, S., Roncato, S., \& Umiltì, C. (1982). Transformation processes upon the visual code. Perception \& Psychophysics, 31, 13-25.

NOTE

1. By using the term "rate of normalization," we simply intend to describe the increase in RT as a function of the difference in orientation between the upper and the lower letters. We do not mean to imply that a mental rotation has actually taken place.

(Manuscript received May 31, 1983;

Revision accepted for publication November $21,1983$. ) 